Supplementary information for manuscript

\title{
Organic aerosol components derived from 25 AMS datasets across Europe using a newly developed ME-2 based source apportionment strategy
}

Crippa, M. ${ }^{1}$, Canonaco, F. ${ }^{1}$, Lanz, V. A. ${ }^{1}$, Äijälä, M. ${ }^{2}$, Allan, J. D. ${ }^{3}$, Carbone, S. ${ }^{4}$, Capes, G. ${ }^{3}$, Dall'Osto, M. ${ }^{5}$, Day, D. A. ${ }^{6}$, DeCarlo, P. F. ${ }^{1, * *}$, Di Marco, C. F. ${ }^{7}$, Ehn, M. $^{2}$, Eriksson, A. ${ }^{8}$, Freney, E. ${ }^{9}$, Hildebrandt Ruiz, L. ${ }^{10, * * *}$, Hillamo, R. ${ }^{4}$, Jimenez, J.L. $^{6}$, Junninen, H. ${ }^{2}$, Kiendler-Scharr, A. ${ }^{11}$, Kortelainen, A.-M. ${ }^{12}$, Kulmala, M. $^{2}$, Mensah, A. A. ${ }^{11, * * * * *}$, Mohr, C. ${ }^{1, *}$, Nemitz, E. ${ }^{7}$, O'Dowd, C. ${ }^{13}$, Ovadnevaite, J. ${ }^{13}$, Pandis, S. N. ${ }^{14}$, Petäjä, T. ${ }^{2}$, Poulain, L. ${ }^{15}$, Saarikoski, S. ${ }^{4}$, Sellegri, K. ${ }^{9}$, Swietlicki, E. ${ }^{8}$, Tiitta, . $^{16}$, Worsnop, D. R. ${ }^{2,4,16,17}$, Baltensperger, U. ${ }^{1}$, and Prévôt, A. S. H ${ }^{1}$.

[1]\{Laboratory of Atmospheric Chemistry, Paul Scherrer Institute, PSI Villigen, 5232, Switzerland $\}$

[2] \{Department of Physics, P.O. Box 64, 00014, University of Helsinki, Finland \}

[3]\{National Centre for Atmospheric Science, School of Earth, Atmospheric \& Environmental Sciences, The University of Manchester, Oxford Road, Manchester, M13 9PL, UK\}

[4]\{Air Quality Research, Finnish Meteorological Institute, P.O. Box 503, FI-00101 Helsinki, Finland

[5]\{Institut de Ciències del Mar, CSIC Pg Marítim de la Barceloneta 37-49 08003, Barcelona, Spain $\}$

[6] \{Cooperative Institute for Research in Environmental Sciences (CIRES), USA \}

[7]\{Centre for Ecology and Hydrology, Bush Estate, Penicuik, Midlothian, EH26 0QB, UK\}

[8]\{Division of Nuclear Physics, University of Lund, SE-221 00 Lund, Sweden\} [9]\{Laboratoire de Météorologie Physique, CNRS-Université Blaise Pascal, UMR6016, 63117, Clermont Ferrand, France\}

[10] \{Center for Atmospheric Particle Studies, Carnegie Mellon University, 5000 Forbes Ave., Pittsburgh, PA, 15213, US \}

[11]\{ Institut für Energie- und Klimaforschung: Troposphäre (IEK 8), Forschungszentrum Jülich GmbH, Jülich, Germany

[12]\{Department of Physics, Univ. of Eastern Finland, P.O. Box 1627, 70211 Kuopio, Finland

[13]\{School of Physics \& Centre for Climate \& Air Pollution Studies, National University of Ireland Galway, Ireland \}

[14]\{Institute of Chemical Engineering Sciences (ICE-HT) Foundation for Research and Technology Hellas (FORTH), Patras, 26504, Greece

[15]\{Leibniz Institute for Tropospheric Research, Permoserstr 15, 04318, Leipzig, Germany

[16] \{Department of Environmental Science, Univ. of Eastern Finland, P.O. Box 1627, 70211 Kuopio, Finland

[17]\{Aerodyne Research, Inc. Billerica, MA, USA\} 
[*] \{now at: Department of Atmospheric Sciences, University of Washington, Seattle WA 98195, USA

$\left[{ }^{* *}\right]$ \{now at: Department of Civil, Architectural, and Environmental Engineering and Department of Chemistry, Drexel University, Philadelphia, PA, USA, 19104\}

$[* * *]$ nnow at: The University of Texas at Austin, McKetta Department of Chemical Engineering, Austin, TX, 78712, US \}

[****] \{now at: ETH Zurich, Institute for Atmospheric and Climate Science, Switzerland $\}$

Correspondence to: A.S.H. Prévôt (andre.prevot@psi.ch) 


\section{SI-1 Average chemical composition}

Table SI-1: Mean concentrations (in $\mu \mathrm{g} / \mathrm{m}^{3}$ ) of AMS chemical components for the EMEP/EUCAARI campaigns.

\begin{tabular}{|c|c|c|c|c|c|c|c|c|c|c|c|c|c|c|c|}
\hline \multirow{2}{*}{ Site } & \multicolumn{5}{|c|}{ Spring 2008} & \multicolumn{5}{|c|}{ Fall 2008} & \multicolumn{5}{|c|}{ Spring 2009} \\
\hline & Org & $\mathrm{NO}_{3}^{-}$ & $\mathrm{SO}_{4}{ }^{2-}$ & $\mathrm{NH}_{4}^{+}$ & $\mathrm{Chl}^{-}$ & Org & $\mathrm{NO}_{3}^{-}$ & $\mathrm{SO}_{4}{ }^{2-}$ & $\mathrm{NH}_{4}^{+}$ & Chl' $^{-}$ & Org & $\mathrm{NO}_{3}^{-}$ & $\mathrm{SO}_{4}{ }^{2-}$ & $\mathrm{NH}_{4}^{+}$ & $\mathrm{ChI}^{-}$ \\
\hline Barcelona & & & & & & & & & & & 8.20 & 3.60 & 2.70 & 1.60 & 0.24 \\
\hline Cabauw & 4.20 & 2.50 & 1.50 & 1.70 & 0.06 & & & & & & 1.20 & 2.20 & 1.00 & 1.00 & 0.15 \\
\hline Finokalia & 2.60 & 0.08 & 5.00 & 1.50 & 0.01 & & & & & & 1.40 & 0.05 & 1.40 & 0.40 & 0.01 \\
\hline Helsinki & & & & & & & & & & & 2.90 & 0.90 & 2.90 & 0.80 & 0.04 \\
\hline Hyytiälä & & & & & & 0.80 & 0.10 & 0.50 & 0.20 & 0.01 & 1.40 & 0.20 & 1.40 & 0.40 & 0.01 \\
\hline Jungfraujoch & 0.66 & 0.27 & 0.41 & 0.21 & 0.01 & & & & & & & & & & \\
\hline K-Puszta & & & & & & 5.30 & 2.00 & 2.70 & 1.60 & 0.10 & & & & & \\
\hline Mace Head & 0.90 & 0.20 & 0.80 & 0.30 & 0.02 & & & & & & 0.80 & 0.60 & 0.40 & 0.30 & 0.05 \\
\hline Melpitz & 6.90 & 0.70 & 2.40 & 0.90 & 0.02 & 3.90 & 3.00 & 1.70 & 1.40 & 0.10 & 1.40 & 3.10 & 1.10 & 1.40 & 0.12 \\
\hline Montseny & & & & & & & & & & & 3.50 & 3.80 & 1.50 & 2.00 & 0.11 \\
\hline Payerne & & & & & & 5.40 & 2.70 & 1.70 & 1.60 & 0.03 & 4.10 & 3.90 & 1.10 & 1.70 & 0.08 \\
\hline Puijo & & & & & & 0.90 & 0.10 & 0.30 & 0.10 & 0.01 & & & & & \\
\hline Puy de Dome & & & & & & 1.76 & 0.82 & 1.73 & 1.52 & 0.02 & 0.57 & 0.74 & 0.32 & 0.56 & 0.03 \\
\hline San Pietro Capofiume & 3.80 & 2.90 & 1.40 & 1.40 & 0.16 & & & & & & & & & & \\
\hline Vavihill & & & & & & 3.70 & 3.20 & 1.60 & 1.60 & 0.16 & 2.60 & 1.80 & 0.90 & 0.80 & 0.09 \\
\hline Chilbolton & & & & & & & & & & & 2.50 & 3.00 & 1.50 & 1.50 & 0.29 \\
\hline Harwell & & & & & & 3.21 & 3.12 & 1.72 & 1.57 & 0.11 & & & & & \\
\hline
\end{tabular}




\section{SI-2 Source apportionment results}

Table SI-2: Comparison of PMF and ME-2 results. Constrained factors in ME-2 are highlighted in red. The reference spectra were taken from Crippa et al. (2013) for the HOA and COA sources $(a$-value $=0.05)$, and from $\mathrm{Ng}$ et al. (2011) for the BBOA component $(a$-value=0.3). For the PMF solutions of Barcelona, San Pietro Capofiume, Cabauw and Finokalia refer to Mohr et al. (2012), Saarikoski et al. (2012), Paglione et al. (2013), and Hildebrandt et al., (2010;2011), respectively.

\begin{tabular}{|c|c|c|c|c|c|c|c|}
\hline \multirow{2}{*}{ Site } & & \multicolumn{2}{|c|}{ April/May 2008} & \multicolumn{2}{|c|}{ Sep/Oct 2008} & \multicolumn{2}{|c|}{ Feb/Mar 2009} \\
\hline & & PMF & ME2 & PMF & $\begin{array}{r}\text { ME2 } \\
\end{array}$ & \begin{tabular}{|l|} 
PMF \\
\end{tabular} & ME2 \\
\hline Barcelona & ES & & & & & $\begin{array}{c}\text { HR-PMF:LV-OOA, SV } \\
\text { OOA, HOA, BBOA, } \\
\text { COA }\end{array}$ & $\begin{array}{c}\text { HOA,BBOA,COA,S } \\
\text { V-OOA, LV-OOA }\end{array}$ \\
\hline Cabauw & NL & $\begin{array}{l}\text { LV-OOA, SV- } \\
\text { OOA,Hulis, HOA }\end{array}$ & $\begin{array}{c}\text { HOA, BBOA,SV- } \\
\text { OOA, LV-OOA }\end{array}$ & & & $\begin{array}{c}\text { LV-OOA, SV-OOA, } \\
\text { HOA, BBOA }\end{array}$ & $\begin{array}{l}\text { HOA, BBOA, SV- } \\
\text { OOA, LV-OOA }\end{array}$ \\
\hline Finokalia & GR & LV-OOA, SV-OOA & $\begin{array}{c}\text { SV-OOA, LV- } \\
\text { OOA, HOA,MSA }\end{array}$ & & & $\begin{array}{c}\text { OOA, LOA (OB-OA), } \\
\text { AOA }\end{array}$ & - \\
\hline Helsinki & FI & & & & & $\begin{array}{c}\text { HOA, BBOA, SV- } \\
\text { OOA, LV-OOA }\end{array}$ & $\begin{array}{c}\text { HOA,BBOA, SV- } \\
\text { OOA,LV-OOA }\end{array}$ \\
\hline Hyytiälä & FI & & & LV-OOA, SV-OOA & $\begin{array}{c}\text { HOA,BBOA,SV-OOA, } \\
\text { LV-OOA }\end{array}$ & LV-OOA, SV-OOA & $\begin{array}{c}\text { HOA,BBOA, SV- } \\
\text { OOA, LV-OOA }\end{array}$ \\
\hline Jungfraujoch & $\mathrm{CH}$ & LV-OOA, OOA*, POA & $\begin{array}{c}\mathrm{HOA}, \mathrm{BBOA}, \mathrm{LV}- \\
\mathrm{OOA}\end{array}$ & & & & \\
\hline K-Puszta & HU & & & $\begin{array}{l}\text { SV-OOA, LV-OOA, } \\
\text { HOA, BBOA like }\end{array}$ & $\begin{array}{c}\text { HOA,BBOA, SV-OOA, } \\
\text { LV-OOA }\end{array}$ & & \\
\hline Mace Head & IR & $\begin{array}{c}\text { HOA,MSA,SV-OOA, } \\
\text { LV-OOA }\end{array}$ & $\begin{array}{l}\text { HOA,BBOA,MSA, } \\
\text { SV-OOA,LV-OOA }\end{array}$ & & & $\mid \begin{array}{c}\mathrm{HOA}, \mathrm{BBOA} \text { like, MSA } \\
\text { like, LV-OOA }\end{array}$ & $\begin{array}{c}\mathrm{HOA}, \mathrm{BBOA}, \mathrm{MSA}, \mathrm{L} \\
\text { V-OOA }\end{array}$ \\
\hline Melpitz & DE & LV-OOA, SV-OOA & $\begin{array}{c}\text { HOA,SV-OOA,LV- } \\
\text { OOA } \\
\end{array}$ & $\begin{array}{c}\text { HOA like,LV-OOA, } \\
\text { SV-OOA }\end{array}$ & $\begin{array}{l}\text { HOA,BBOA,SV- } \\
\text { OOA,LV-OOA }\end{array}$ & $\begin{array}{c}\text { POA, LV-OOA, SV- } \\
\text { OOA }\end{array}$ & $\begin{array}{c}\text { HOA, BBOA,SV- } \\
\text { OOA,LV-OOA }\end{array}$ \\
\hline Montseny & ES & & & & & \begin{tabular}{|c|} 
POA, SV-OOA, LV- \\
OOA (HR-PMF: HOA, \\
BBOA, OOA) \\
\end{tabular} & $\begin{array}{l}\mathrm{HOA}, \mathrm{BBOA}, \mathrm{LV}- \\
\text { OOA }\end{array}$ \\
\hline Payerne & $\mathrm{CH}$ & & & OOA, POA & $\begin{array}{c}\text { HOA,BBOA, SV-OOA, } \\
\text { LV-OOA }\end{array}$ & $\begin{array}{c}\text { POA,SV-OOA,LV- } \\
\text { OOA }\end{array}$ & $\begin{array}{c}\text { HOA,BBOA, SV- } \\
\text { OOA, LV-OOA }\end{array}$ \\
\hline Puijo & $\mathrm{FI}$ & & & $\mathrm{OOA}$ & $\mathrm{HOA}, \mathrm{OOA}$ & & \\
\hline Puy de Dome & FR & & & SV-OOA, LV-OOA & $\begin{array}{l}\text { HOA,BBOA,SV- } \\
\text { OOA,LV-OOA }\end{array}$ & LV-OOA, SV-OOA & $\begin{array}{l}\text { HOA, BBOA,SV- } \\
\text { OOA, LV OOA }\end{array}$ \\
\hline $\begin{array}{l}\text { San Pietro } \\
\text { Capofiume }\end{array}$ & IT & $\begin{array}{c}\text { LV-OOA, SV-OOA } \\
\text { (HR PMF: HOA, } \\
\text { BBOA, N-OA, OOA-a, } \\
\text { OOA-b, OOA-c) } \\
\end{array}$ & $\begin{array}{l}\text { HOA,BBOA,SV- } \\
\text { OOA, LV-OOA }\end{array}$ & & & & \\
\hline Vavihill & SE & & & $\mathrm{HOA}, \mathrm{BBOA}, \mathrm{LV}-\mathrm{OOA}$ & HOA,BBOA,LV-OOA & $\begin{array}{c}\text { HOA,SV-OOA,LV- } \\
\text { OOA }\end{array}$ & $\begin{array}{l}\text { HOA,BBOA,SV- } \\
\text { OOA,LV-OOA }\end{array}$ \\
\hline Chilbolton & UK & & & & & $\begin{array}{c}\text { HOA,BBOA,SV- } \\
\text { OOA,LV-OOA }\end{array}$ & $\begin{array}{c}\text { HOA,BBOA,SV- } \\
\text { OOA,LV-OOA }\end{array}$ \\
\hline Harwell & UK & & & & & POA,LV-OOA & $\begin{array}{c}\text { HOA,BBOA,SV- } \\
\text { OOA,LV-OOA }\end{array}$ \\
\hline
\end{tabular}


Table SI-3: Correlation matrix of the OA factors and their tracers

Table SI-3 reports the correlation $\left(R^{2}\right)$ between the time series of the OA sources and available tracers. HOA correlates with black carbon measurements, BBOA with the fraction of organic at mass 60 , while SV-OOA and LV-OOA are compared with $\mathrm{NO}_{3}$ and $\mathrm{SO}_{4}$, respectively.

\begin{tabular}{l|c|c|c|c}
\hline $\mathbf{R}^{2}$ & HOA vs. BC & BBOA vs. org60 & SV-OOA vs. NO3 & LV-OOA vs. SO4 \\
\hline Barcelona & 0.7 & 0.42 & 0.37 & 0.61 \\
\hline Cabauw 2008 & 0.68 & 0.81 & 0.27 & 0.5 \\
\hline Cabauw 2009 & 0.43 & 0.82 & 0.75 & 0.67 \\
\hline Chilbolton spring 2009 & 0.82 & 0.79 & 0.22 & 0.24 \\
\hline Finokalia 2008 & - & - & 0.11 & 0.74 \\
\hline Helsinki 2009 & - & 0.76 & 0.07 & 0.58 \\
\hline Hyytiälä 2008 & 0.72 & 0.9 & 0.29 & 0.36 \\
\hline Hyytiälä 2009 & 0.66 & 0.65 & 0.11 & 0.74 \\
\hline Jungfraujoch 2008 & - & 0.71 & 0.79 & - \\
\hline K-Puszta 2008 & - & 0.87 & 0.19 & 0.64 \\
\hline Mace Head 2008 & - & 0.74 & - & - \\
\hline Mace Head 2009 & - & 0.98 & 0.6 & 0.81 \\
\hline Melpitz spring 2008 & - & - & 0.22 & 0.58 \\
\hline Melpitz fall 2008 & 0.33 & 0.8 & - & 0.5 \\
\hline Melpitz spring 2009 & 0.74 & 0.8 & 0.27 & 0.34 \\
\hline Montseny & - & 0.64 & - & 0.74 \\
\hline Payerne fall 2008 & - & 0.4 & 0.14 & 0.57 \\
\hline Payerne spring 2009 & - & 0.87 & 0.12 & 0.54 \\
\hline Puijo & - & - & - & 0.5 \\
\hline Puy de Dome fall 2008 & 0.77 & 0.41 & 0.57 & 0.54 \\
\hline Puy de Dome spring 2009 & - & 0.97 & 0.93 & 0.11 \\
\hline San Pietro Capofiume & - & 0.81 & 0.49 & 0.11 \\
\hline Vavihill fall 2008 & - & 0.23 & - & 0.58 \\
\hline Vavihill spring 2009 & & 0.14 & 0.41 \\
\hline
\end{tabular}




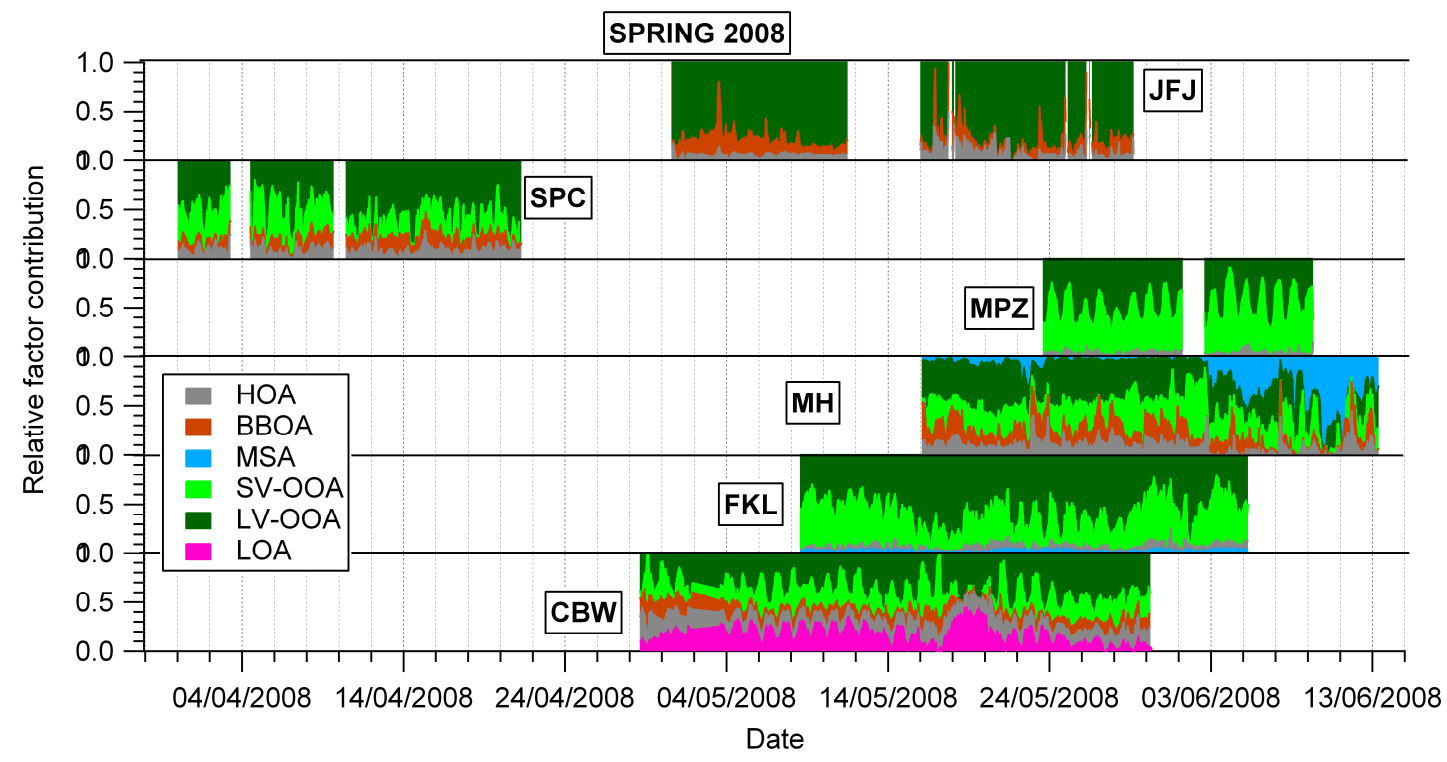

Fig. SI-2.1: Temporal variation of the relative contributions of organic aerosol sources during the spring 2008 campaigns. 


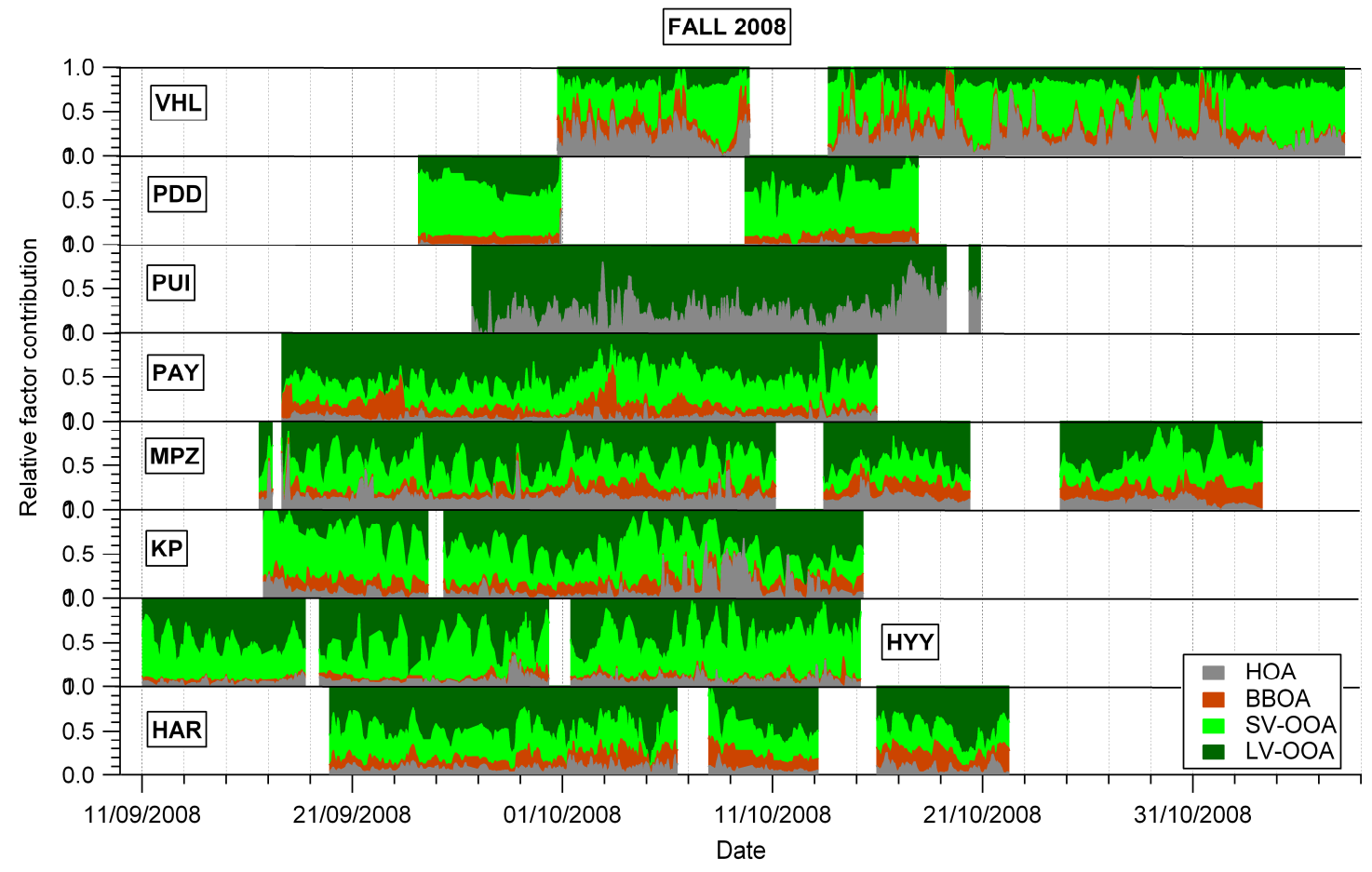

Fig. SI-2.2: Temporal variation of the relative contributions of organic aerosol sources during the fall 2008 campaigns. 
SPRING 2009

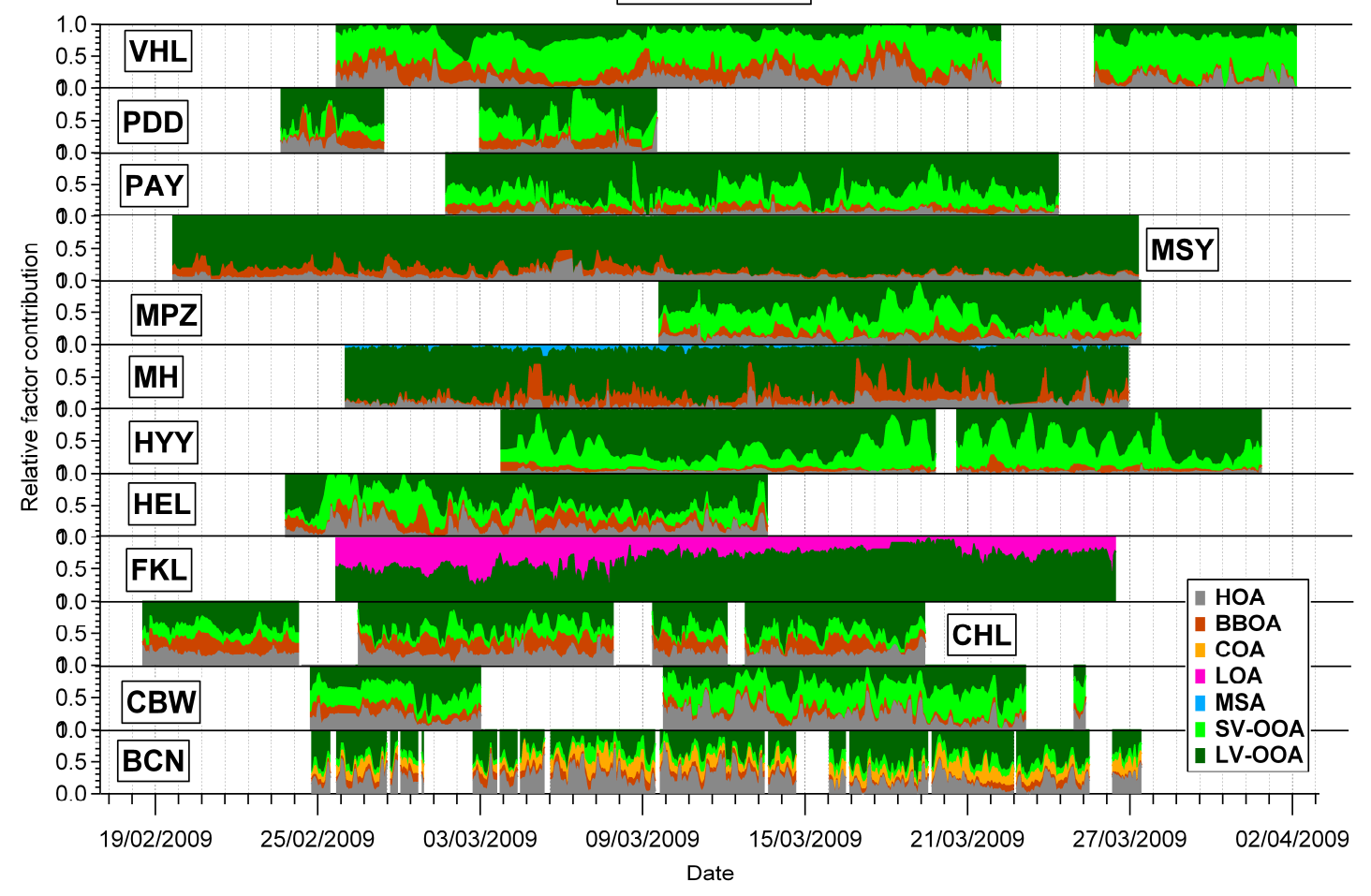

Fig. SI-2.3: Temporal variation of the relative contributions of organic aerosol sources during the spring 2009 campaigns. 


\section{SI-3 Comparison of results from different source apportionment methods}

In this section the comparison between source apportionment solutions retrieved with our standardized method and UMR/HR-PMF results available for some field campaigns is reported. This analysis shows that our source apportionment procedure produces quite comparable results with UMR/HR-PMF in the cases of Montseny and Barcelona (Figs. SI-3.1 and SI-3.2). Bigger discrepancies are indeed observed for the Cabauw case (Fig. SI-3.3) because our solution additionally includes a BBOA factor compared to the work of Paglione et al. (2013).
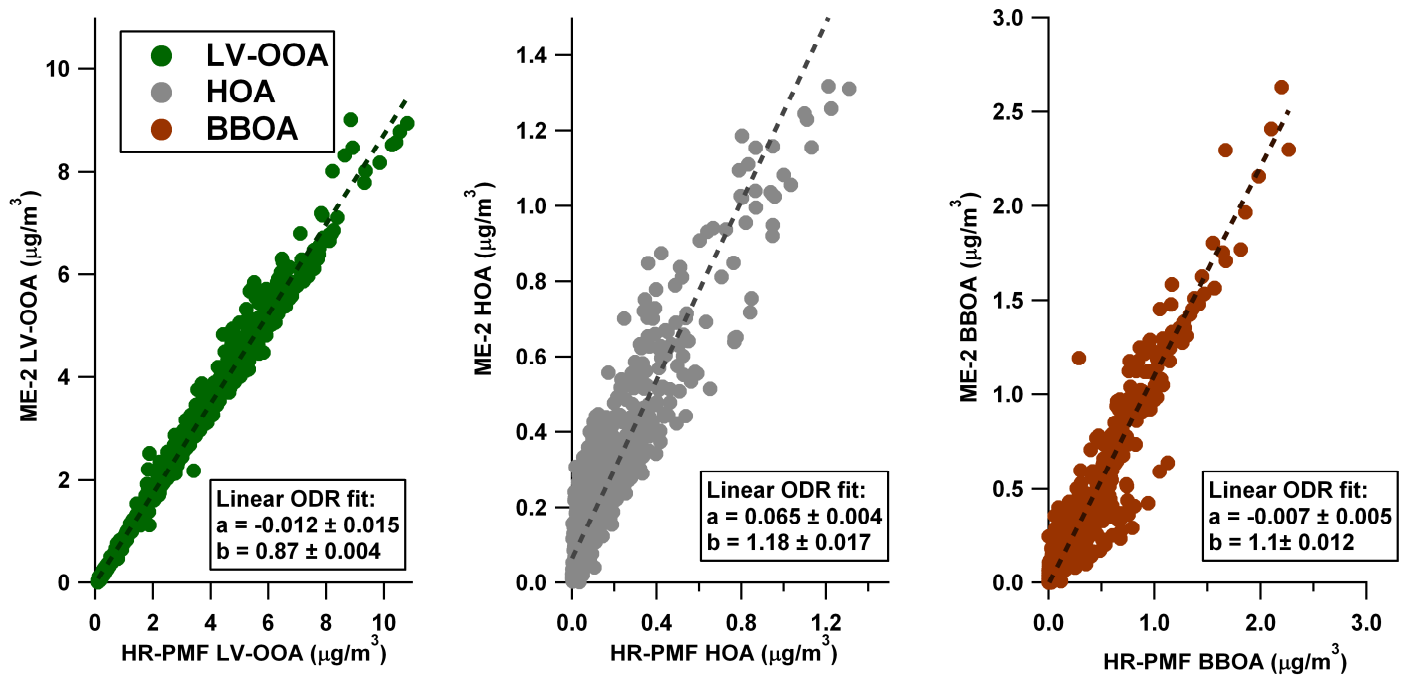

Fig. SI-3.1: Time series comparison of OA sources between the ME-2 solution and the HR-PMF solution for the Montseny spring 2009 campaign. 

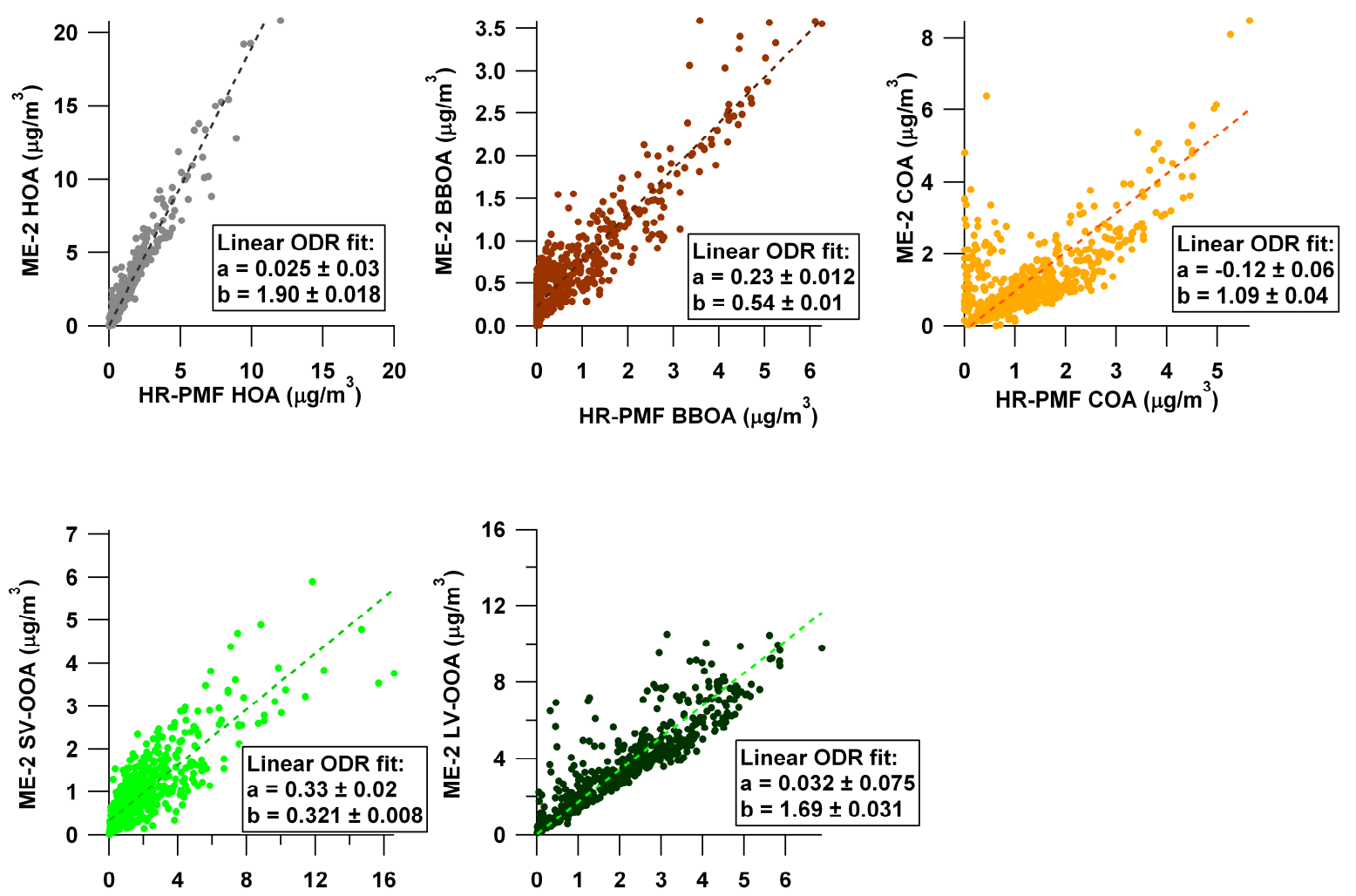

HR-PMF SV-OOA $\left(\mu \mathrm{g} / \mathrm{m}^{3}\right)$

HR-PMF LV-OOA $\left(\mu \mathrm{g} / \mathrm{m}^{3}\right)$

Fig. SI-3.2: Time series comparison of OA sources between the ME-2 solution and the HR-PMF solution for the Barcelona spring 2009 campaign (Mohr et al., 2012). 

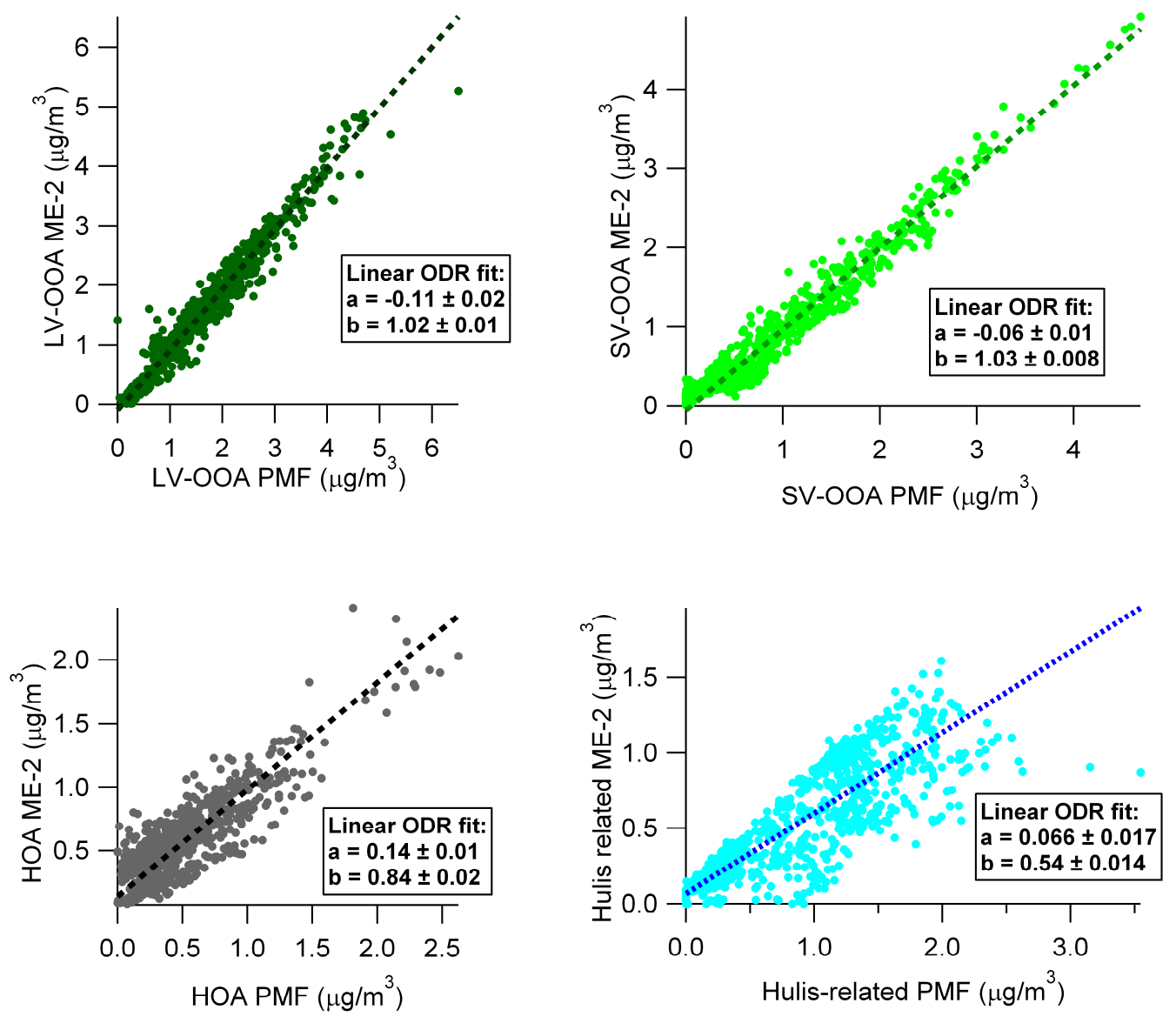

Fig. SI-3.3: Time series comparison of OA sources between the ME-2 solution and the PMF solution for the Cabauw spring 2008 campaign (Paglione et al., 2013). 


\section{SI-4 Reference mass spectra}

Figure SI-4 represents the MSA mass spectrum obtained for the Mace Head spring 2008 data, which was then chosen as reference spectrum to be constrained in the ME-2 approach for the Finokalia 2008 and Mace Head 2009 campaigns (Ovadnevaite et al., in prep). Typical peaks of MSA fragmentation in the AMS contribute to this spectrum, such as $m / z$ 15, 45, 65, 78, 79, 96 (Zorn et al., 2008). However this MS contains some interferences from sea salt at $m / z, 58$ and 60 .

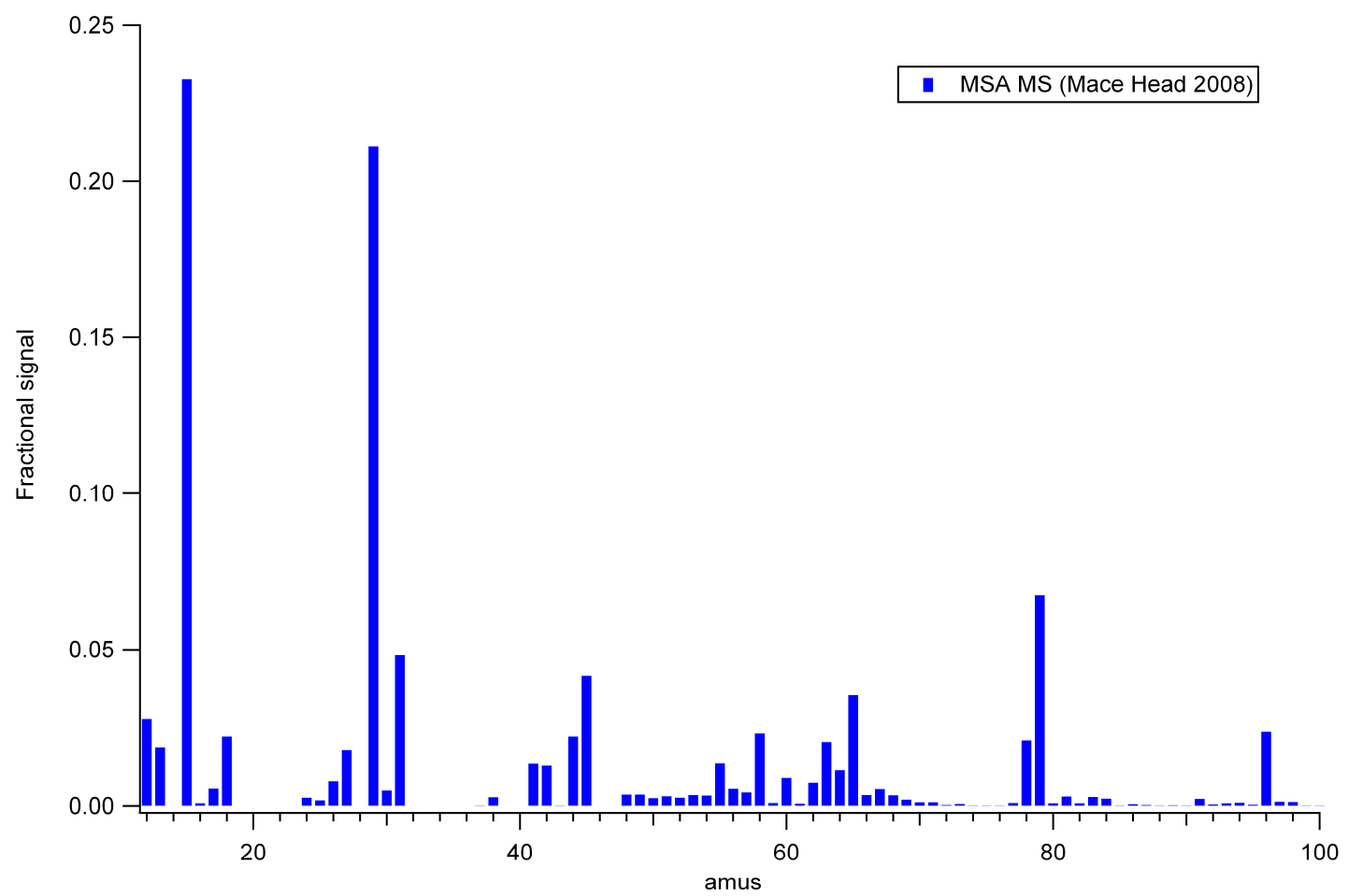

Figure SI-4: MSA MS obtained for the Mace Head 2008 campaign, chosen as reference MSA MS for all the marine sites. 


\section{References}

Crippa, M., DeCarlo, P. F., Slowik, J. G., Mohr, C., Heringa, M. F., Chirico, R., Poulain, L., Freutel, F., Sciare, J., Cozic, J., Di Marco, C. F., Elsasser, M., José, N., Marchand, N., Abidi, E., Wiedensohler, A., Drewnick, F., Schneider, J., Borrmann, S., Nemitz, E., Zimmermann, R., Jaffrezo, J.-L., Prévôt, A. S. H., and Baltensperger, U.: Wintertime aerosol chemical composition and source apportionment of the organic fraction in the metropolitan area of Paris, Atmos. Chem. Phys., 13, 961-981, 2013.

Hildebrandt, L., Engelhart, G. J., Mohr, C., Kostenidou, E., Lanz, V. A., Bougiatioti, A., DeCarlo, P. F., Prevot, A. S. H., Baltensperger, U., Mihalopoulos, N., Donahue, N. M., and Pandis, S. N.: Aged organic aerosol in the Eastern Mediterranean: the Finokalia Aerosol Measurement Experiment-2008, Atmos. Chem. Phys., 10, 4167-4186, 2010.

Hildebrandt, L., Kostenidou, E., Lanz, V. A., Prevot, A. S. H., Baltensperger, U., Mihalopoulos, N., Laaksonen, A., Donahue, N. M., and Pandis, S. N.: Sources and atmospheric processing of organic aerosol in the Mediterranean: insights from aerosol mass spectrometer factor analysis, Atmos. Chem. Phys., 11, 12499-12515, 2011.

Mohr, C., DeCarlo, P. F., Heringa, M. F., Chirico, R., Slowik, J. G., Richter, R., Reche, C., Alastuey, A., Querol, X., Seco, R., Peñuelas, J., Jiménez, J. L., Crippa, M., Zimmermann, R., Baltensperger, U., and Prévôt, A. S. H.: Identification and quantification of organic aerosol from cooking and other sources in Barcelona using aerosol mass spectrometer data, Atmos. Chem. Phys., 12, 1649-1665, 2012.

Ng, N. L., Canagaratna, M. R., Jimenez, J. L., Zhang, Q., Ulbrich, I. M., and Worsnop, D. R.: Real-time methods for estimating organic component mass concentrations from aerosol mass spectrometer data, Environ. Sci. Technol., 45, 910-916, 2011.

Paglione, M., Kiendler-Scharr, A., Mensah, A. A., Finessi, E., Giulianelli, L., Sandrini, S., Facchini, M. C., Fuzzi, S., Schlag, P., Piazzalunga, A., Tagliavini, E., Henzing, J. S., and and Decesari, S.: Identification of humic-like substances (HULIS) in oxygenated organic aerosols using NMR and AMS factor analyses and liquid chromatographic techniques, Atmos. Chem. Phys. Discuss., 13, 17197-17252, 2013.

Saarikoski, S., Carbone, S., Decesari, S., Giulianelli, L., Angelini, F., Canagaratna, M., Ng, N. L., Trimborn, A., Facchini, M. C., Fuzzi, S., Hillamo, R., and Worsnop, D.: Chemical characterization of springtime submicrometer aerosol in Po Valley, Italy, Atmos. Chem. Phys., 12, 8401-8421, 2012.

Zorn, S. R., Drewnick, F., Schott, M., Hoffmann, T., and Borrmann, S.: Characterization of the South Atlantic marine boundary layer aerosol using an aerodyne aerosol mass spectrometer, Atmos. Chem. Phys., 8, 4711-4728, 2008. 